\title{
Line defects in epitaxial silicon films grown at $560^{\circ} \mathrm{C}$
}

\author{
K. Petter ${ }^{a, *}$ D. Eyidi ${ }^{b}$ M. Stöger-Pollach ${ }^{b}$ I. Sieber ${ }^{\text {a }}$ P. Schubert-Bischoff ${ }^{\text {a }}$ B. Rau ${ }^{\text {a }}$ \\ A. T. Tham ${ }^{\text {c,d }}$ P. Schattschneider ${ }^{b}$ S. Gall ${ }^{a}$ K. Lips ${ }^{a}$ W. Fuhs ${ }^{\text {a }}$ \\ ${ }^{\mathrm{a}}$ Hahn-Meitner-Institut, Kekuléstr. 5, D-12489 Berlin, Germany \\ ${ }^{\mathrm{b}}$ Technische Universität Wien, Wiedner Hauptstr. 8-10, A-1040 Wien, Austria \\ ${ }^{\mathrm{c}}$ Fritz-Haber-Institut der Max-Planck-Gesellschaft, Faradayweg 4-6, D-14195 Berlin, Germany \\ ${ }^{\mathrm{d}}$ present adress: Endress+Hauser GmbHEGCo.KG, Oderstr. 54, D-14513 Teltow, Germany
}

\begin{abstract}
We present an investigation of line defects in epitaxially grown silicon layers using Secco defect etching and transmission electron microscopy (TEM). $1 \mu \mathrm{m}$ thick layers were deposited onto Si (100) wafers at a substrate temperature of $560^{\circ} \mathrm{C}$ using electron cyclotron resonance chemical vapour deposition (ECRCVD). Defect etching reveals a variety of etch pits related to extended defects. A detailed analysis of the orientations and shapes of etch pits related to line defects is carried out. Using this information it is then possible to assign different types of etch pits to line defects observed by TEM. The investigations show, that one type of defect are extended dislocations parallel to $<112>$, while the direction of two other types are $<110>$ as well as $<314>$, a direction uncommon for line defects in silicon.
\end{abstract}

Key words:

Silicon, epitaxy, defect etching

$P A C S$ :

\section{Introduction}

Extended defects like dislocations, stacking faults or grain boundaries in silicon are known to have a detrimental effect on the minority carrier lifetime [1]. Therefore a variety of groups investigated the structure of dislocations [2] and their effect on the performance of solar cells prepared from silicon wafers [3,4]. In epitaxially grown silicon layers (epi-Si) a similar behavior was observed [5]. Studies on the structure of dislocations [6], however, revealed that they may differ from what is usually found in wafer material [7]. In this work we present an investigation of line defects in silicon layers epitaxially grown by electron cyclotron resonance chemical vapour deposition (ECRCVD) at tempera-

\footnotetext{
* Hahn-Meitner-Institut, Kekuléstr. 5, D-12489 Berlin, Germany

Tel: +49-30-8062-1396, Fax: +49-30-8062-1333, email: petter@hmi.de
}

tures below $600^{\circ} \mathrm{C}$. Structural defects in the layers are uncovered by defect etching using Secco etch [8]. The use of a scanning electron microscope (SEM) makes it possible to analyse the etch pits in detail and distinguish different types by their shape, crystallographic orientation and size as reported in [9]. Here, we will concentrate on elliptical etch pits related to line defects. We will discuss the properties of the etch pits and show how this information can be used to determine the orientation of the underlying defect. This makes it possible to clearly assign the etch pits to line defects observed in TEM investigations.

\section{Experimental}

The silicon films were deposited using ECRCVD at a substrate temperature of $560^{\circ} \mathrm{C}$ onto slightly boron doped (100) oriented FZ-Silicon wafers with a resistiv- 


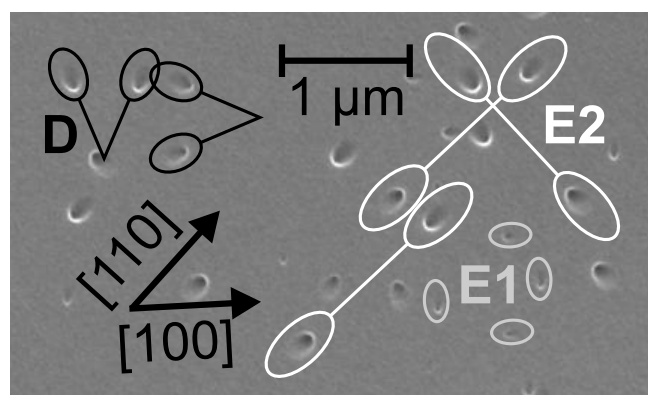

Fig. 1. Topview SEM image showing the different types of elliptical etch pits observed after Secco etching. The lines connecting $\mathrm{E} 2$ and $\mathrm{D}$ pits indicate defect pairs as discussed in the text.

ity of $3 \mathrm{k} \Omega \mathrm{cm}$. The layers are epitaxially grown and approximately $1 \mu \mathrm{m}$ thick. Although the layers were not intentionally doped, they show n-type conductivity with charge carrier concentrations in the range of $10^{16}$ to $10^{17} \mathrm{~cm}^{-3}$ due to contamination with impurities [9]. Structural defects were uncovered by etching the surface of the layers using Secco etch $(50 \%$ $\mathrm{HF}: 0.15 \mathrm{~m} \mathrm{~K}_{2} \mathrm{Cr}_{2} \mathrm{O}_{7}$, mixed 2:1) as has been reported before $[1,3,4]$. In these works the density of dislocations was estimated from the etch pit density observed by optical microscopy after etch times of several minutes. In our case etch times are limited $(\sim 10 \mathrm{~s})$ in order to assure that the thin epitaxial layer is not etched away (bulk etch rate of Secco etch $\sim 1.5 \mu \mathrm{m} / \mathrm{min}[8]$ ). This inevitably leads to smaller etch pits $(<1 \mu \mathrm{m})$, so that the use of an SEM (Hitachi S4100) is necessary. This makes it possible to not only determine etch pit densities as in the case of optical microscopy but also obtain detailed information on the shapes and directions of the etch pits. Total etch pit densities are of the order of $10^{8} \mathrm{~cm}^{-2}$, while the densities of the different types of etch pits vary depending on the deposition conditions [9]. TEM investigations were performed using a FEI TECNAI F20 S-TWIN microscope under multi-, twobeam or weak-beam dark-field conditions. The crosssection samples were prepared in either (100) or (110) planes depending on the defect under investigation.

\section{Results}

The elliptical etch pits found in our samples can be put into three categories (E1, E2, D) as indicated in the overview SEM image of a Secco etched surface of an epi-layer shown in Fig. 1. The properties of the different types of etch pits and their correlation to defects observed in TEM images will now be discussed.

The E1 etch pits are elliptically shaped with their main axis being approximately $200 \mathrm{~nm}$ long and aligned parallel to the $<100>$ directions. The elliptical

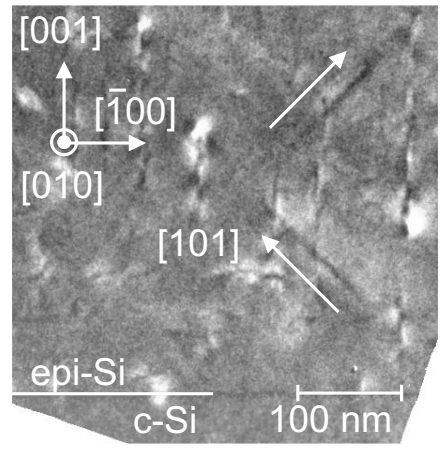

Fig. 2. Cross-section TEM image of defects responsible for the E1 etch pits. The line direction of the defects is $<110>$.

shape is typical for line defects penetrating the surface at some angle. From the direction of the etch pits on the (001) surface we can conclude that the direction of the underlying defects are of the form $\langle 10 \mathrm{z}\rangle$, where $\mathrm{z}$ indicates that the component in growth direction cannot be deduced from this topview image. Crosssection TEM images of a sample with a high density of E1 prepared in a (010) plane can provide this information. In Fig. 2 line defects in $\langle 101\rangle$ directions can be observed, as indicated by the arrows. Note that the vertical lines also visible are due to defects of the same kind which lie in the (100) plane perpendicular to the (010) plane. Since no other defects with directions of the form $\langle 10 z\rangle$ were observed, we deduce, that this defect is the cause for the E1 etch pits.

E2 are elliptical etch pits whose main axis is approximately $300 \mathrm{~nm}$ long and aligned parallel to the $<110>$ directions. This shows that the direction of the underlying defect can be written in the form $\langle 11 \mathrm{z}\rangle$. As indicated in Fig. 1 these etch pits are often found in pairs whose two partners lie on a $<110>$ line. The separation of the two partners, $\mathrm{r}_{\mathrm{E} 2}$, depends on the thickness of the epitaxial layer, $d_{L}$, as shown in Fig. 3 . Note that the vertical line at each data point represents the spread of $\mathrm{r}_{\mathrm{E} 2}$ observed for each sample. The linear dependence observed in Fig. 3 indicates that the defect pairs start to grow in approximately the same depth below the surface. Assuming that this is the epi-Si/c-Si interface, it is possible to get an estimate for the $\mathrm{Z}$ component of the defect orientation. The lines added to the graph give the calculated values of $\mathrm{r}_{\mathrm{E} 2}$ for line defect pairs that start to grow at the epi-Si/c-Si interface into the given directions. It becomes obvious from Fig. 3 that the experimental data are best described assuming a direction of $\langle 112>$. However, the observed values of $\mathrm{r}_{\mathrm{E} 2}$ lie on or slightly below the predicted behaviour for defects with $<112>$ directions.

To get more insight into this behavior, TEM investigations were performed on a sample that exhibits a high E2 density. Fig. 4 shows a TEM image of two defect pairs where both partners lie exactly in $<112>$ 


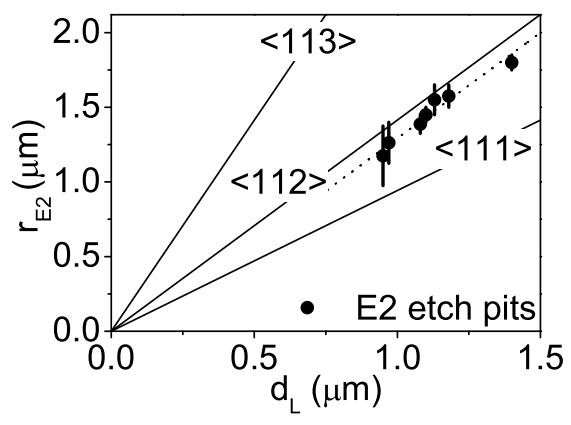

Fig. 3. Experimentally determined separation, $\mathrm{r}_{\mathrm{E} 2}$, (dots) between the partners of the E2 etch pit pairs on the layer thickness, $d_{L}$. The solid lines represent the separation calculated for defect pairs that grow into the given directions starting at the epi-Si/c-Si interface. The dotted line is a fit to determine the average distance of the "kink" to the interface (see text).

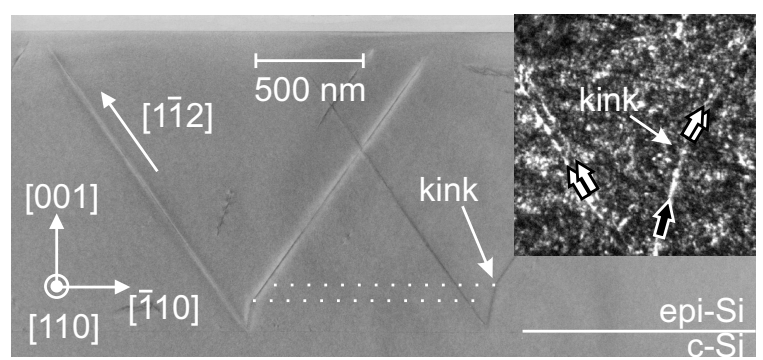

Fig. 4. TEM image of $\mathrm{V}$ shaped defects responsible for the E2 etch pits. The line direction of the defects is $\langle 112\rangle$. The dotted lines indicate the plane parallel to the epi-Si/c-Si interface where the kink is observed for the two defect pairs. The inset shows a more detailed image of the region around the kink.

directions. Near the interface the direction of one of the two line defects is tilted towards the surface normal. The distance from the interface where this "kink" occurs is not fixed but varies from pair to pair as indicated by the two dotted lines. The presence of this kink will therefore result in a slightly smaller $\mathrm{r}_{\mathrm{E} 2}$ than expected for a pure $<112>$ direction. Taking this kink into account, the data in Fig. 3 can be fitted with a linear function (dotted line) from which a mean distance of the kink from the interface of about $230 \mathrm{~nm}$ can be estimated. This is in good agreement with what is found in Fig 4. These defects therefore explain the dependence of $\mathrm{r}_{\mathrm{E} 2}$ on $\mathrm{d}_{\mathrm{L}}$ and also its variations and can be identified as the origin of the E2 etch pits.

To get more insight into the region around the kink, a more detailed image is shown in the inset of Fig. 4. For this image the sample was slightly tilted, revealing that the defect is a single line in the region between the kink and the interface (black arrow), but a set of two parallel lines in the region, where the direction is $\langle 112\rangle$ (white arrows). In the region between the kink and the interface the Burgers vector (b) was determined to be perpendicular to the dislocation line

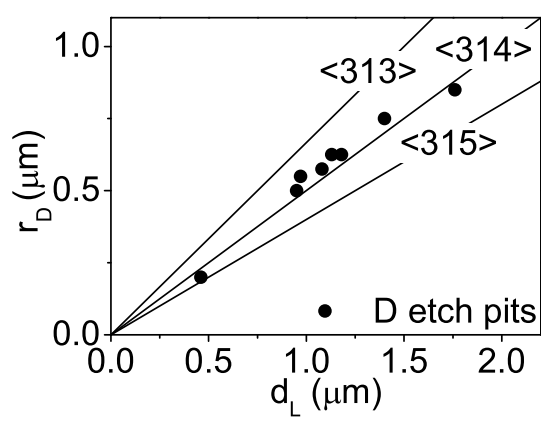

Fig. 5. Dependence of the separation, $\mathrm{r}_{\mathrm{D}}$, between the partners of the D etch pit pairs as a function of the layer thickness, $\mathrm{d}_{\mathrm{L}}$. The lines give the separation calculated for defect pairs that grow into the given directions starting at the epi-Si/c-Si interface.

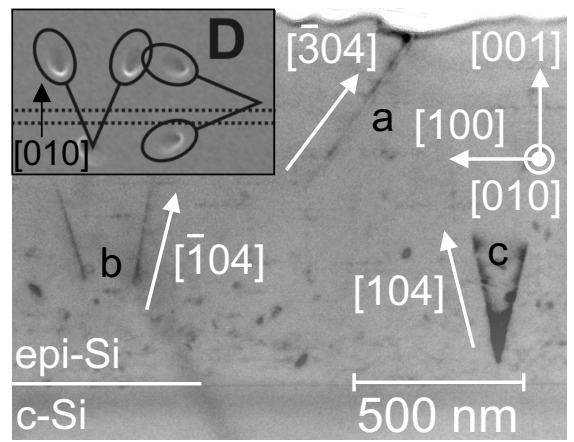

Fig. 6. TEM image of defects responsible for the D etch pits. The line direction of the defects is $\langle 314\rangle$, the projections on the $(010)$ plane are therefore $<304>$ and $<104>$ oriented. The inset shows a detail from Fig. 1 . The dotted lines indicate the boundaries of a (fictitious) TEM sample prepared in the (010) plane.

$\left(b=\frac{1}{2}<110>\right)$ showing that it is a perfect edge dislocation. Above the kink the defect consists of two lines which can therefore be interpreted as two partial dislocations with a stacking fault in between them, known as an extended dislocation [10].

The D etch pits are elliptically shaped, with their main axis being $\sim 300 \mathrm{~nm}$ long and tilted by $\sim 20^{\circ}$ with respect to the $\langle 100\rangle$ direction. From this angle the direction of the underlying defect can be estimated to be of the form $\langle 31 \mathrm{z}\rangle$, which corresponds to an angle of $18.4^{\circ}$ with respect to $<100>$ (the directions $\left.<41 \mathrm{z}\right\rangle$ and $\langle 21 \mathrm{z}\rangle$ would result in an angle of $14^{\circ}$ and $26^{\circ}$, respectively). These etch pits also appear in pairs as indicated in Fig. 1. The separation of the two partners depends on the layer thickness as in the case of E2 (Fig. 5). Therefore, the Z component of the direction of the underlying defect can be determined by the same procedure as in the case of E2. Fig. 5 shows the separations, $\mathrm{r}_{\mathrm{D}}$, calculated for different orientations of the defect. Obviously, the experimental data (dots) are 


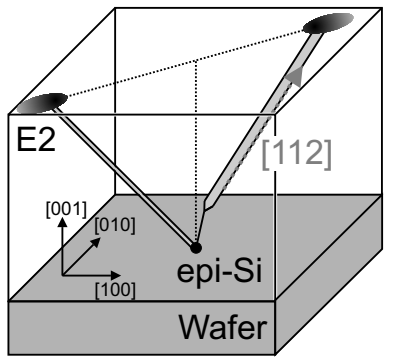

a)

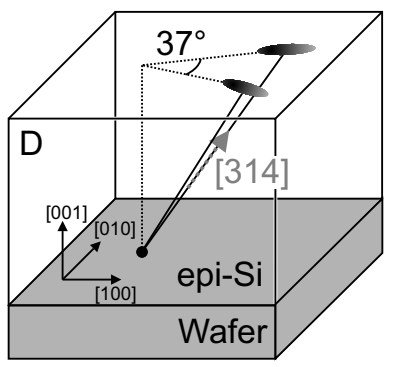

b)
Fig. 7. Schematic drawing of a) E2- and b) D-defects

best described assuming that the orientation of the defects is $<314>$. This is supported by the TEM image shown in Fig. 6 which was taken on a sample prepared in the (010) plane. Since $<314>$ does not lie in a (010) plane and the TEM sample has a the finite thickness only parts of the defect lines are present in the thin slice. For better visualisation the inset shows a detail from Fig. 1, where the dotted lines indicate the boundaries of a (fictitious) TEM sample prepared in a (010) plane. Depending on the orientation of the D pairs, either both or just one partner are present in the sample. In the TEM image (parts of) three defect pairs are visible (a-c). In two cases (b and c) both partners are visible, while the pair "a" is oriented perpendicular to $\mathrm{b}$ and $\mathrm{c}$, so that only one partner is visible. The projections of the direction estimated for the D-Pairs $(<314>)$ onto the $(010)$ plane are indicated by the arrows. These directions agree well with the directions of the defects, so that they can unambiguously be identified as the cause for the D etch pit pairs.

\section{Discussion}

As has been shown in the proceeding paragraph, the E1, E2 and D etch pits can be related to specific defects observed in TEM images. From the evaluation of the data obtained from defect etching and TEM analysis, structural models of the E2 and D defects can be deduced (Fig. 7). For the case of E2 the presented results show, that these defects can be interpreted as being due to extended dislocations with a line direction parallel to $<112>$ (Fig. 7a). This direction is usually not observed for dislocations in bulk silicon [7], however, in epitaxial layers this direction seems to be favourable for dislocations as shown by Prieur et al. [6]. They deposited epitaxial layers onto SIMOX substrates that contained dislocations with a line direction of $\langle 110\rangle$. These dislocations are extended into the epitaxial layer, however, the line direction changed to $<112>$.
The $\mathrm{D}$ defects are defect pairs, whose partners have an orientation of $<314>$ (Fig. $7 \mathrm{~b}$ ). The direction $<314>$ is not a common direction for crystal defects in silicon. However, the fact that $<314>$ lies in a (111) plane just as dislocations along $<110>$ or $<112>$ may be taken as an evidence that these are also dislocations. Further investigation is needed to support this.

Although no detailed investigation of the E1 defects was carried out yet, we associate this defect with the so called line-interstitial defect (LID), which was recently assigned to a photoluminescence (PL) line at $0.88 \mathrm{eV}$ by Mchedlidze et. al. [11]. This PL line is also observed in our samples and its intensity correlates to the density of the E1 etch pits, as described in [9].

Two of the observed defects (E2/D) were shown to have their origin at the epi-Si/c-Si interface. In order to reduce the defect density in the layers, the reason for this behaviour has to be investigated. Possible explanations include a contamination of the surface of the wafer before deposition leading to defect formation at sites where clusters of impurities are present. Another possibility is related to impurities in the layers themselves. As described in [9] the epi-layers contain oxygen and nitrogen in concentrations of $10^{18} \mathrm{~cm}^{-3}$ and above. Incorporation of impurities can result in a variation of the lattice constant compared to a pure crystal. The resulting stress may also lead to the formation of the observed defects. These issues have to be taken into account in our future in order to reduce the defect density.

\section{Conclusion}

We have identified three types of elliptical etch pits with the underlying line defects observed in TEM images. The line orientation of the line defects were shown to be $<110>(\mathrm{E} 1),<112>(\mathrm{E} 2)$ and $<314>$ (D). The E2 defects were identified to be extended dislocations. Future work will include the question wether the E1 defects are indeed LIDs, as indicated by PL measurements, as well as determining the nature of the $\mathrm{D}$ defects with their uncommon line direction $(<314>)$.

\section{Acknowledgements}

We would like to thank Kerstin Jacob, Sabrina Kreutzmann and Brunhilde Rabe for sample preparation. This work has been partially supported by the European Commission within the framework of the METEOR project (ENK5-CT-2001-00543). 


\section{References}

[1] A. Kurtz, S. Kulin, B. Averbach, Phys. Rev. 101 (1956) 1285 .

[2] M. Sato, K. Hiraga, K. Sumino, Jpn. J. Appl. Phys 19 (1980) L155.

[3] K. Pauls, K. Mitchell, W. Chesarek, Conference Record of the Twenty Third IEEE Photovoltaic Specialists Conference (1993) 209.

[4] T. Ciszek, T. Wang, R. Burrows, T. Bekkedahl, M. Symko, J. Webb, Solar Energy Materials and Solar Cells 41/42 (1996) 61.

[5] L. Oberbeck, R. Bergmann, J. Appl. Phys. 88 (5) (2000) 3015 .

[6] E. Prieur, C. Guilhalmenc, J. Härtwig, M. Ohler, A. Garcia, B. Aspar, J. Appl. Phys. 80 (4) (1996) 2113.

[7] K. Sumino, Dislocations in silicon crystals, in: J. Nishizawa (Ed.), Semiconductor Technology, Vol. 6, North-Holland, Amsterdam, Netherlands, 1983, p. 23.

[8] F. Secco d'Aragona, J. Electrochem. Soc. 119 (1972) 948.

[9] K. Petter, I. Sieber, B. Rau, S. Brehme, K. Lips, W. Fuhs, Thin Solid Films in Press.

[10] S. Amelinckx, in: F. R. N. Nabarro (Ed.), Dislocations in Solids, Vol. 2, 1979, p. 67.

[11] T. Mchedlidze, S. Binetti, A. Le Donne, M. Suezawa, S. Pizzini, phys. stat. sol. (c) 2 (2005) 1807. 